\title{
ARTICLE
}

Clinical Study

\section{A randomised phase II trial of S-1 plus cisplatin versus vinorelbine plus cisplatin with concurrent thoracic radiotherapy for unresectable, locally advanced non-small cell lung cancer: WJOG5008L}

\author{
Tomonari Sasaki ${ }^{1}$, Takashi Seto ${ }^{1}$, Takeharu Yamanaka ${ }^{2}$, Naonobu Kunitake $^{1}$, Junichi Shimizu ${ }^{3}$, Takeshi Kodaira $^{3}$, Makoto Nishio ${ }^{4}$ \\ Takuyo Kozuka ${ }^{4}$, Toshiaki Takahashi ${ }^{5}$, Hideyuki Harada ${ }^{5}$, Naruo Yoshimura ${ }^{6}$, Shinichi Tsutsumi ${ }^{6}$, Hiromoto Kitajima ${ }^{7}$, Masaaki Kataoka $^{7}$, \\ Yukito Ichinose ${ }^{1}$, Kazuhiko Nakagawa ${ }^{8}$, Yasumasa Nishimura ${ }^{8}$, Nobuyuki Yamamoto ${ }^{5}$ and Yoichi Nakanishi ${ }^{9}$
}

\begin{abstract}
BACKGROUND: Cisplatin-based chemoradiotherapy is the standard treatment for unresectable, locally advanced non-small-cell lung cancer (NSCLC). This trial evaluated two experimental regimens that combine chemotherapy with concurrent radiotherapy. METHODS: Eligible patients with unresectable stage III NSCLC were randomised to either the SP arm (S-1 and cisplatin) or VP arm (vinorelbine and cisplatin), with early concurrent thoracic radiotherapy of $60 \mathrm{~Gy}$, comprising $2 \mathrm{~Gy}$ per daily fraction. The primary endpoint was the overall survival rate at 2 years (2-year overall survival (OS)) (Study ID: UMIN000002420).

RESULTS: From September 2009 to September 2012, 112 patients were enroled. Of the 108 eligible patients, the 2-year OS was $75.6 \%(80 \%$ confidence interval $(\mathrm{Cl}), 67-82 \%)$ in the SP arm and $68.5 \%(80 \% \mathrm{Cl}: 60-76 \%)$ in the VP arm. The hazard ratio (HR) for death between the two arms was 0.85 (0.48-1.49). The median progression-free survival was 14.8 months for the SP arm and 12.3 months for the VP arm with an HR of $0.92(0.58-1.44)$. There were four treatment-related deaths in the SP arm and five in the VP arm.

CONCLUSIONS: The null hypotheses for 2-year OS were rejected in both arms. The West Japan Oncology Group will employ the SP arm as the investigational arm in a future phase III study.
\end{abstract}

British Journal of Cancer (2018) 119:675-682; https://doi.org/10.1038/s41416-018-0243-2

\section{INTRODUCTION}

Non-small-cell lung cancer (NSCLC) is one of the leading causes of cancer death worldwide. ${ }^{1}$ The standard treatment for patients with unresectable, locally advanced NSCLC (LA-NSCLC) is chemotherapy with concurrent radiotherapy, although the optimal chemotherapeutic agents remain undefined. Recently, chemotherapeutic regimens have advanced from the second to third generation. ${ }^{2,3}$

Yamamoto et al. ${ }^{3}$ published the results of a phase III trial (WJTOG0105) that was conducted to compare third-generation chemotherapy (carboplatin with paclitaxel, or cisplatin with irinotecan) with second-generation chemotherapy (cisplatin plus mitomycin plus vindesine: MVP) both in conjunction with concurrent thoracic radiotherapy (TRT), and reported no significant difference in overall survival (OS). Segawa et al. $^{2}$ also published the results of a phase III clinical trial that compared concurrent TRT with docetaxel plus cisplatin (DP) with concurrent TRT with MVP. There was a trend towards improved survival in the DP arm compared with the MVP arm $(p=0.059)$. However, these previous trials failed to show that third-generation regimens were significantly superior to second-generation regimens.

Alternatively, the West Japan Oncology Group (WJOG) has developed another chemotherapeutic regimen consisting of cisplatin and S-1 for patients with LA-NSCLC. S-1 (TS- $1^{\oplus}$; Taiho Pharmaceutical Co. Ltd., Tokyo, Japan) is a third-generation oral fluoropyrimidine anticancer agent that combines tegafur, gimeracil, and oteracil potassium in a molar ratio of 1.0:0.4:1.0. ${ }^{4}$ We conducted a single-arm phase II study, WJTOG3706, to evaluate the efficacy and safety of SP with concurrent TRT. The OS rate at 2 years (2-year OS) was $70 \%$ with a response rate of $84 \%$. These are promising results and appear to be superior to other second-generation or thirdgeneration regimens employed in previous clinical trials. ${ }^{5}$

\footnotetext{
${ }^{1}$ National Kyushu Cancer Center, 3-1-1 Notame, Minami-ku, Fukuoka 811-1395, Japan; ${ }^{2}$ Yokohama City University, 3-9 Fukuura, Kanazawa-ku, Yokohama 236-0004, Japan; ${ }^{3}$ Aichi Cancer Center Hospital, 1-1 Kanokoden, Chikusa-ku, Nagoya 464-8681, Japan; ${ }^{4}$ Cancer Institute Hospital of Japanese Foundation for Cancer Research, 3-8-31, Ariake, Koto-ku, Tokyo 135-8550, Japan; ${ }^{5}$ Shizuoka Cancer Center, 1007 Shimonagakubo, Nagaizumi-cho, Sunto-gun, Shizuoka 411-8777, Japan; ${ }^{6}$ Osaka City University Hospital, 1-5-7 Asahimachi, Abeno-ku, Osaka 545-8586, Japan; ${ }^{7}$ Shikoku Cancer Center, 160 Kou, Minamiumemoto-machi, Matsuyama City, Ehime 791-0280, Japan; ${ }^{8}$ Kindai University Hospital, 377-2 Ohnohigashi, Osaka-Sayama 589-8511, Japan and ${ }^{9}$ Kyushu University Hospital, 3-1-1 Maidashi, Higashi-ku, Fukuoka 812-8582, Japan

Correspondence: Takashi Seto (tseto@nk-cc.go.jp)

Presented in part at the Annual Meeting of the American Society of Clinical Oncology, 1 June 2015.
}

Received: 4 January 2018 Revised: 18 July 2018 Accepted: 2 August 2018

Published online: 12 September 2018 
At present, in conjunction with concurrent TRT, vinorelbine plus cisplatin (VP), or DP and carboplatin plus paclitaxel are thirdgeneration regimens frequently used in patients with unresectable stage III LA-NSCLC. Radiotherapy was given concurrently during the second and third cycles of chemotherapy (four cycles in total) in overseas phase II studies, with reports that the median survival time ranged from 16 to 17 months. ${ }^{6,7}$ In Japan, Sekine et al. ${ }^{8}$ reported one-week cessation of ongoing radiotherapy and use of docetaxel as additional chemotherapy following concurrent radiotherapy using the VP regimen. However, no prospective studies have been conducted to evaluate concurrent radiotherapy and additional chemotherapy in a single VP regimen without suspending radiotherapy. Moreover, unlike SP regimens, no reproducible data are available regarding VP regimens. To select the optimal VP regimen in Japan, we therefore considered it necessary to give concurrent radiotherapy and additional chemotherapy in a single VP regimen on the basis of the regimen proposed by Sekine et al., ${ }^{8}$ followed by an evaluation of this therapeutic approach.

As a result of the preceding study (WJTOG0105), carboplatin plus paclitaxel is now considered a standard chemoradiotherapy regimen for LA-NSCLC in Japan. However, cisplatin plus etoposide is standard chemoradiotherapy outside Japan, and a direct comparison with the cisplatin-based regimen is required. To evaluate which treatment regimen, SP or VP, would be best as an investigational arm in a future study, we decided to conduct a phase II randomised study and compare these regimens.

\section{PATIENTS AND METHODS}

Patient selection

Patients with histologically or cytologically proven NSCLC with unresectable, locally advanced disease were assessed for eligibility. The definition of locally advanced disease is described in detail in the protocol, although in brief it constitutes unresectable stage III disease. Eligible patients also needed to meet the following criteria: no prior history of chemotherapy or TRT or surgery; and patients who can be treated with radiotherapy according to the protocol. Other eligibility requirements included age of 20-74 years, Eastern Cooperative Oncology Group performance status of $0-1$, and adequate organ function.

For staging, all patients underwent chest X-ray (CXR), computed tomography (CT) of the thorax and abdomen, and either brain $\mathrm{CT}$ or brain magnetic resonance imaging. A radioisotopic bone scan or positron emission tomography was also performed on all patients.

All patients provided written informed consent before enrolment in this study. The protocol was designed in accordance with the Declaration of Helsinki and ethical guidelines for clinical research and was approved by the institutional review boards at all participating institutions.

\section{Randomisation}

Eligible patients were randomly assigned to the SP arm or the VP arm at the WJOG data centre. Randomisation was achieved by a minimisation method and stratified by disease stage (stage IIIA or IIIB), sex (male or female), histology (adenocarcinoma or nonadenocarcinoma) and institution. Patients and investigators were not masked to treatment.

\section{Procedures}

Treatment comprised concurrent chemoradiotherapy and subsequent consolidation chemotherapy.

In the SP arm, patients received oral S-1 $\left(80 \mathrm{mg} / \mathrm{m}^{2}\right)$ in two divided doses daily after meals on days 1-14 and cisplatin $\left(60 \mathrm{mg} / \mathrm{m}^{2}\right)$ as an intravenous (i.v.) infusion on day 1 . The dose of S-1 was determined according to body surface area (BSA) as follows: BSA $<1.25 \mathrm{~m}^{2}, 80 \mathrm{mg}$ per day; BSA $1.25 \mathrm{~m}^{2}$ to $<1.50 \mathrm{~m}^{2}$,
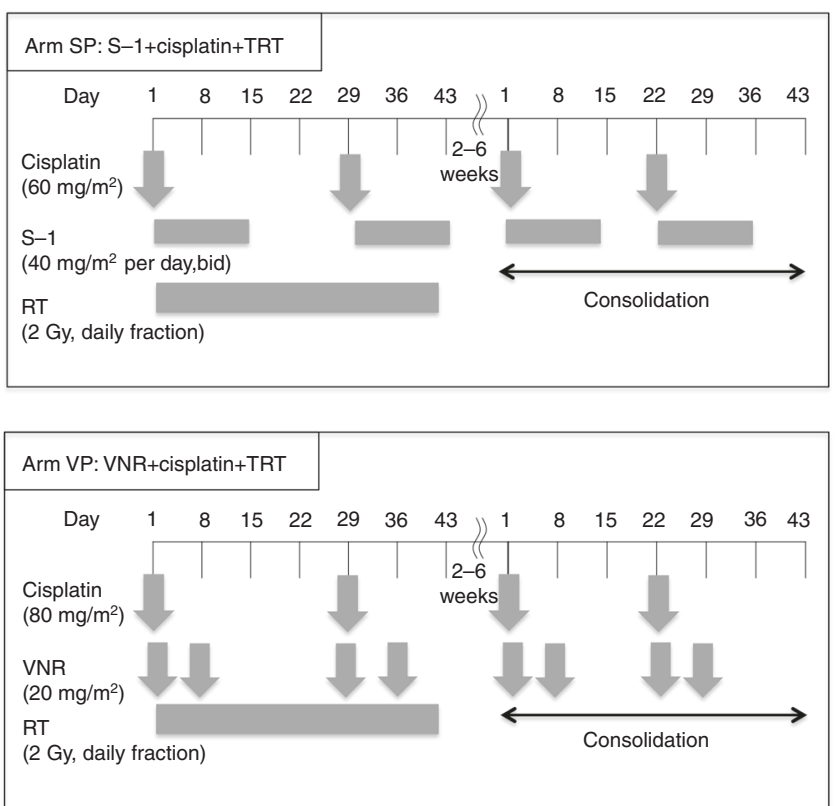

Fig. 1 Treatment schedule. The treatment schedule of each arm. SP arm: S-1 plus cisplatin plus thoracic radiotherapy (TRT), VP arm: vinorelbine (VNR) plus cisplatin plus thoracic radiotherapy

$100 \mathrm{mg}$ per day; and BSA $1.5 \mathrm{~m}^{2}$ or higher, $120 \mathrm{mg}$ per day. Combination chemotherapy with SP was repeated twice, with a 4week interval, concurrently with TRT. In the VP arm, patients received vinorelbine $20 \mathrm{mg} / \mathrm{m}^{2}$ on days 1 and 8 , and cisplatin 80 $\mathrm{mg} / \mathrm{m}^{2}$ on day 1 . Chemotherapy with VP was repeated every 4 weeks for two cycles, concurrently with TRT. Two to six weeks after the completion of the concurrent TRT, two further cycles of the same SP or VP regimen were administered every 3 weeks as consolidation chemotherapy (Fig. 1).

\section{Radiotherapy}

All patients were treated with a linear accelerator photon beam of 6-10 MV from day 1 . The primary tumour and involved nodal disease received $60 \mathrm{~Gy}$ in $2 \mathrm{~Gy}$ fractions over a period of 6 weeks. In this protocol, three-dimensional (D) treatment planning systems were acquired, and $40 \mathrm{~Gy}$ of prophylactic mediastinal irradiation was administered. The doses were calculated assuming tissue homogeneity with correction for lung tissue. The initial $40 \mathrm{~Gy} / 20$ fractions were delivered to clinical target volume 1 (CTV1), and the final $20 \mathrm{~Gy} / 10$ fractions were given to a reduced volume defined as clinical target volume 2 (CTV2). CTV1 included the primary tumour, ipsilateral hilum, and mediastinal nodal areas from the paratracheal (no. 2) to subcarinal lymph nodes (no. 7). For the primary tumours and involved lymph nodes with a short-axis diameter of $1 \mathrm{~cm}$ or larger, a margin of at least $0.5 \mathrm{~cm}$ was added. The contralateral hilum was not included in CTV1. The supraclavicular areas were not treated routinely, but were treated when the supraclavicular nodes were involved. CTV2 included only the primary tumour and the involved lymph nodes, with a margin of 0.5 to $1 \mathrm{~cm}$. The spinal cord was excluded from the fields for CTV2 by appropriate methods, such as the oblique opposing method. An appropriate planning target volume margin and leaf margin were added for CTV1 and CTV2.

TRT was interrupted at the onset of grade 4 haematologic toxicity, grade 3 to 4 oesophagitis or dermatitis, pyrexia of $38^{\circ} \mathrm{C}$, or a decrease in the partial pressure of arterial oxygen of 10 Torr or more, compared with that measured before the initiation of TRT. If a rest period of more than 2 weeks was required, the patient was withdrawn from the study. 
In this trial, radiotherapy data for all patients were submitted to the WJOG data centre and reviewed by the members of the radiotherapy committee (RC). The quality assurance (QA) assessment is described in the protocol. Individual cases were reviewed at a QA review meeting, which was held after each cohort of 15-20 patients had been registered. All data items including pretreatment images (contrast-enhanced chest $C T$, ${ }^{18}$ F-fluorodeoxyglucose positron emission tomography/CT) and radiotherapy data (beam data, target volume, dose distribution, dose-volume histogram) submitted to the data centre were reviewed and evaluated in the meeting.

Evaluation of efficacy and toxicity

All eligible patients who received any treatment at all were considered assessable for response and toxicity. CXR, complete blood counts and blood chemistry tests were repeated once a week during the treatment period. Thoracic CT was performed once a month during the treatment period. After the conclusion of treatment, thoracic CT was performed every 3 months and other imaging examinations were performed when recurrence was suspected. Responses $\geq$ were evaluated according to Response Evaluation Criteria in Solid Tumour, version 1.0. During evaluation of the response, extramural review was conducted. Adverse events were evaluated according to the Common Terminology Criteria for Adverse Events (v3.0). OS was defined as the time from registration until death from any cause. Progression-free survival (PFS) was defined as the time between enrolment and disease progression, death or last known follow-up.

Statistical analysis

The full analysis set included all patients who received the study treatment at least once, were observed for survival, and did not violate the eligibility criteria. The safety analysis set was defined as all patients who received the study treatment at least once. The primary endpoint of this trial was comparison of the 2-year OS rate between the SP arm and VP arm at 2 years (2-year OS rate). This trial was designed to test the null hypothesis that the true 2-year OS rate is less than or equal to a threshold of $50 \%$ versus the alternative hypothesis that the true 2-year OS rate is $\geq 65 \%$. With this design, the one-sided $a$ is 0.10 . The $80 \%$ two-sided confidence interval (CI) for 2 -year OS rate was used to test the null hypothesis. The $\mathrm{Cl}$ was calculated using Greenwood's formula. The patient assignment period was 2 years, and the follow-up period was 2 years. In view of the possibility of variance inflation owing to censoring, the sample size was set at 110 . Baseline characteristics were compared among the treatment groups using the Kruskal-Wallis test for continuous variables and Fisher's exact test for discrete variables. Rates of specific toxicities and treatment delivery were compared between the groups using Fisher's exact test. Survival curves were estimated by the Kaplan-Meier method. The hazard ratios (HRs) were estimated using the Cox proportional hazards method. All statistical analyses were performed using the SAS version 9.1.3 software. We also evaluated OS, PFS, treatment completion rate, and safety as secondary endpoints.

\section{RESULTS}

Four treatment-related deaths (TRDs) were reported in the period between December 2009 and February 2011, and these occurred up to the assignment of the seventy-third patient. Therefore, we suspended registration and checked the details of all randomised patients to assess the safety of treatment regimens. The WJOG Data and Safety Monitoring Committee advised consultation with the WJOG RC about radiotherapy compliance in all patients. The WJOG RC collected each patient's irradiation planning data and did not find an association between poor protocol compliance and TRD. However, it was found that patients who died of radiation pneumonitis had interstitial changes on pretreatment chest CT but not on CXR. Consequently, in August 2011 we decided to continue this trial following the recommendations of the WJOG DSMC, in which the exclusion criteria were updated to exclude patients who had interstitial pneumonia, pulmonary fibrosis or severe emphysema on chest CT images, or who had obstructive pneumonia, active infection (e.g. those who had a fever of at least $38^{\circ} \mathrm{C}$ and who used non-steroidal anti-inflammatory drugs to treat fever), and other serious complications (such as gastrointestinal haemorrhage and cardiac disease).

\section{Patient characteristics}

Between September 2009 and September 2012, a total of 112 patients were registered for the study, and 56 patients were allocated to each arm. Of the total, four patients did not receive the protocol treatment because they became ineligible based on the protocol criteria after registration (two patients) or refused to participate (two patients) (Fig. S1).

The safety and antitumour effects of the treatments were eventually assessed on the basis of the data obtained from 108 enroled patients. There were no statistically significant differences between the arms in terms of patient characteristics (Table 1).

\section{Treatment administered}

During the concurrent phase, $70.4 \%$ of patients in the SP arm and $48.1 \%$ in the VP arm received full cycles of radiotherapy and chemotherapy without any dose reduction within the phase ( $p=$ 0.031). Radiotherapy of $60 \mathrm{~Gy}$ was completed in 102 patients. The main reasons for radiotherapy interruption in all treated patients were fever (9.3\%) in the SP arm and fever (43.4\%), neutropenia $(32.1 \%)$, and leukopenia (24.5\%) in the VP arm. In the consolidation phase, $59.3 \%$ and $44.4 \%$ in the SP arm and the VP arm, respectively, received the two scheduled courses of therapy. Interruption of chemotherapy was more common in the VP arm than in the SP arm during both the concurrent and consolidation phases. Of all treated patients, $7.4 \%$ of patients in the SP arm had a chemotherapy dose reduction, predominantly because of neutropenia (3.7\%), thrombocytopenia (3.7\%), and nonhaematologic toxicities (3.7\%) and $37.0 \%$ of patients in the VP arm had a chemotherapy dose reduction, predominantly because of neutropenia (27.7\%), leukopenia (16.6\%), and nonhaematologic toxicities (14.8\%). Overall, the treatment completion rates were $51.9 \%$ and $29.6 \%$ in the SP and VP arms, respectively $(p=0.031)$ (Table S1).

\section{Toxicity}

Table 2 shows the all-grade and severe toxicities (grades 3-5) including nine patients with TRDs. With regard to grade 3-5 toxicities, leukopenia, neutropenia, and febrile neutropenia were more common in the VP arm than in the SP arm. Alternatively, grade 3-4 thrombocytopenia, oesophagitis, and diarrhoea tended to be more common in the SP arm than in the VP arm.

The cause of TRD was radiation pneumonitis in three patients and bleeding in one of the four patients in the SP arm: radiation pneumonitis in one patient, pneumonia in two patients, and bleeding in two of the five patients in the VP arm. The clinical course of the patients who died of pneumonitis and bleeding are presented in detail in the Supplementary results. QA results from radiotherapy of all patients showed neither improper protocol implementation nor deviation was directly associated with TRDs.

The relationship between the lung V20 measurement and the grade of radiation pneumonitis is shown in Fig. 2. The risk of radiation pneumonitis grades $2-5$ was related to a V20 of $30 \%$ or more $(p=0.048)$. 
Table 1. Baseline demographic and patients characteristics

\begin{tabular}{|c|c|c|c|}
\hline & $\mathrm{SP}(n=54)$ & $\operatorname{VP}(n=54)$ & $P$ value \\
\hline Gender, $n$ (\%) & & & 1 \\
\hline Male & $42(77.8)$ & $43(79.6)$ & \\
\hline Female & $12(22.2)$ & $11(20.4)$ & \\
\hline Age, median (range) & $60(39-73)$ & $62(37-74)$ & 0.073 \\
\hline Stage, $n(\%)$ & & & 1 \\
\hline IIIA & $22(40.7)$ & $22(40.7)$ & \\
\hline IIIB & $32(59.3)$ & $32(59.3)$ & \\
\hline Primary site & & & 0.478 \\
\hline Right upper lobe & $28(51.9)$ & $21(38.9)$ & \\
\hline Right middle lobe & $0(0.0)$ & $2(3.7)$ & \\
\hline Right lower lobe & $2(3.7)$ & $3(5.6)$ & \\
\hline Left upper lobe & $18(33.3)$ & $23(42.6)$ & \\
\hline Left lower lobe & $3(5.6)$ & $4(7.4)$ & \\
\hline Others & $3(5.6)$ & $1(1.9)$ & \\
\hline Histology, n (\%) & & & $\begin{array}{l}0.92 \text { (adeno } \\
\text { versus non- } \\
\text { adeno) }\end{array}$ \\
\hline Adenocarcinoma & $30(55.6)$ & $30(55.6)$ & \\
\hline Squamous cell carcinoma & $17(31.5)$ & $17(31.5)$ & \\
\hline $\begin{array}{l}\text { Adenosquamous cell } \\
\text { carcinoma }\end{array}$ & $1(1.9)$ & $0(0.0)$ & \\
\hline Large-cell carcinoma & $2(3.7)$ & $1(1.9)$ & \\
\hline Others & $4(7.4)$ & $6(11.1)$ & \\
\hline Smoking status, $n(\%)$ & & & 1 \\
\hline Never & $6(11.1)$ & $7(13.0)$ & \\
\hline Ever & $48(88.9)$ & $47(87.0)$ & \\
\hline PS, $n(\%)$ & & & 0.694 \\
\hline 0 & $34(63.0)$ & $31(57.4)$ & \\
\hline 1 & $20(37.0)$ & $23(42.6)$ & \\
\hline Complication, $n$ (\%) & & & 0.335 \\
\hline Absent & $29(53.7)$ & $23(42.6)$ & \\
\hline Present & $25(46.3)$ & $31(57.4)$ & \\
\hline Lung V20 (\%), median (range) & $\begin{array}{l}23.5 \\
(13.1-34.7)\end{array}$ & $\begin{array}{l}25.2 \\
(11.4-34.7)\end{array}$ & 0.306 \\
\hline
\end{tabular}

SP cisplatin plus S-1, VP cisplatin plus vinorelbine, $P S$ performance status

\section{Efficacy}

The objective response rates were $76.9 \%$ (95\% confidence interval (Cl): 63.2-87.5) and $80.8 \%(95 \% \mathrm{Cl}$ : 67.5-90.4) in the SP and VP arms, respectively (Table S2). No efficacy data were obtained from two patients in both arms and all of these results are based on the analysis of 52 patients. There was no statistical difference between these arms. The OS and PFS curves are shown in Fig. 3. Most of the patients were observed for more than 2 years, and 52 patients died. The median follow-up time for the censored patients was 31.9 months (interquartile range: $27.7-47.7$ months), and the 2-year PFS rate for all treated patients was $26.7 \%(95 \% \mathrm{Cl}$ : 18-35\%). The median survival time, the median PFS time, and 2year OS in the SP arm were 40.9 months, 14.8 months, and $75.6 \%$ (80\% Cl: $67-82 \%)$, respectively. The corresponding values in the VP arm were 39.0 months, 12.3 months, and $68.5 \%$ (80\% Cl: $60-76 \%)$. The lower limit of the $\mathrm{Cl}$ for 2-year OS in both arms exceeded the threshold of $50 \%$. However, there was no statistically significant difference in terms of OS between the two arms (HR $=0.85 ; 95 \%$ $\mathrm{Cl}: 0.48-1.49 ; p=0.57)$. Subset analyses show that the OS was not significantly different when various factors are taken into

\begin{tabular}{|c|c|c|c|c|}
\hline \multirow[t]{2}{*}{ Adverse events, $n$ (\%) } & \multicolumn{2}{|l|}{$\mathrm{SP}(n=54)$} & \multicolumn{2}{|l|}{ VP $(n=54)$} \\
\hline & All grades & Grade $3-5$ & All grades & Grade 3-5 \\
\hline \multicolumn{5}{|l|}{ Haematologic toxicities } \\
\hline Leukopenia & $52(96.3)$ & $22(40.7)$ & $54(100.0)$ & $43(79.6)$ \\
\hline Neutropenia & $48(88.9)$ & $18(33.3)$ & $51(94.4)$ & $41(75.9)$ \\
\hline Thrombocytopenia & $23(42.6)$ & $5(9.3)$ & $12(22.2)$ & $2(3.7)$ \\
\hline Anaemia & $43(79.6)$ & $14(25.9)$ & $48(88.9)$ & $15(27.8)$ \\
\hline Febrile neutropenia & $5(9.3)$ & $5(9.3)$ & $9(16.7)$ & $9(16.7)$ \\
\hline \multicolumn{5}{|c|}{ Non-haematologic toxicities } \\
\hline AST increase & $15(27.8)$ & $0(0.0)$ & $15(27.8)$ & $2(3.7)$ \\
\hline ALT increase & $23(42.6)$ & $0(0.0)$ & $25(46.3)$ & $4(7.4)$ \\
\hline $\begin{array}{l}\text { Total bilirubin } \\
\text { increase }\end{array}$ & $14(25.9)$ & $0(0.0)$ & $8(14.8)$ & $0(0.0)$ \\
\hline Nausea & $42(77.8)$ & $2(3.7)$ & $41(75.9)$ & $2(3.7)$ \\
\hline Vomiting & $10(18.5)$ & $1(1.9)$ & $13(24.1)$ & $0(0.0)$ \\
\hline Anorexia & $46(85.2)$ & $7(13.0)$ & $48(88.9)$ & $8(14.8)$ \\
\hline Fatigue & $37(68.5)$ & $2(3.7)$ & $39(72.2)$ & $2(3.7)$ \\
\hline Oesophagitis & $36(66.7)$ & $2(3.7)$ & $40(74.1)$ & $0(0.0)$ \\
\hline Mucositis & $10(18.5)$ & $0(0.0)$ & $9(16.7)$ & $0(0.0)$ \\
\hline Diarrhoea & $19(35.2)$ & $3(5.6)$ & $9(16.7)$ & $0(0.0)$ \\
\hline Creatinine increase & $15(27.8)$ & $0(0.0)$ & $28(51.9)$ & $0(0.0)$ \\
\hline Hyponatremia & $39(72.2)$ & $10(18.5)$ & $35(64.8)$ & $4(7.4)$ \\
\hline Pneumonitis & $13(24.1)$ & $5(9.3)$ & $11(20.4)$ & $4(7.4)$ \\
\hline Bleeding & $1(1.9)$ & $1(1.9)$ & $2(3.7)$ & $2(3.7)$ \\
\hline $\begin{array}{l}\text { Injection site } \\
\text { reaction }\end{array}$ & $1(1.9)$ & $0(0.0)$ & $13(24.1)$ & $0(0.0)$ \\
\hline
\end{tabular}

consideration (Fig. 4). There was also no statistically significant difference in PFS between the arms (HR $=0.92$; 95\% Cl: 0.58-1.44; $p=0.70$ ).

Pattern of recurrence

In this trial, disease recurred in 35 patients in the SP arm and 38 patients in the VP arm. Sites of initial recurrence are shown in Table S3 stratified by the arms. Among the 35 patients with recurrence in the SP arm, in-field relapse was observed in 17 patients $(49 \%, 12$ without and 5 with relapse outside of radiation fields). Distant metastases were the first site of failure in 23 patients. Meanwhile, among the 38 patients with recurrence in the VP arm, in-field relapse was observed in 28 patients (74\%, 19 without and 9 with relapse outside of radiation fields). Distant metastases were the first site of the failure in 19 patients. After the first relapse, 25 and 30 patients in the SP arm and VP arm, respectively, received second-line or further chemotherapy.

\section{DISCUSSION}

This randomised phase II trial was undertaken to evaluate two chemotherapy regimens (SP versus VP) concurrently performed with TRT in patients with unresectable LA-NSCLC. No significant difference between the arms was found. The levels of efficacy and toxicity in each arm were comparable to those seen previous phase II trials. The null hypothesis was rejected in both arms, suggesting that the findings of the present study may be useful in conducting a WJOG-sponsored phase III study, with carboplatin plus paclitaxel as the standard of care. However, in the present study, the frequency of TRDs due to radiotherapy was slightly 


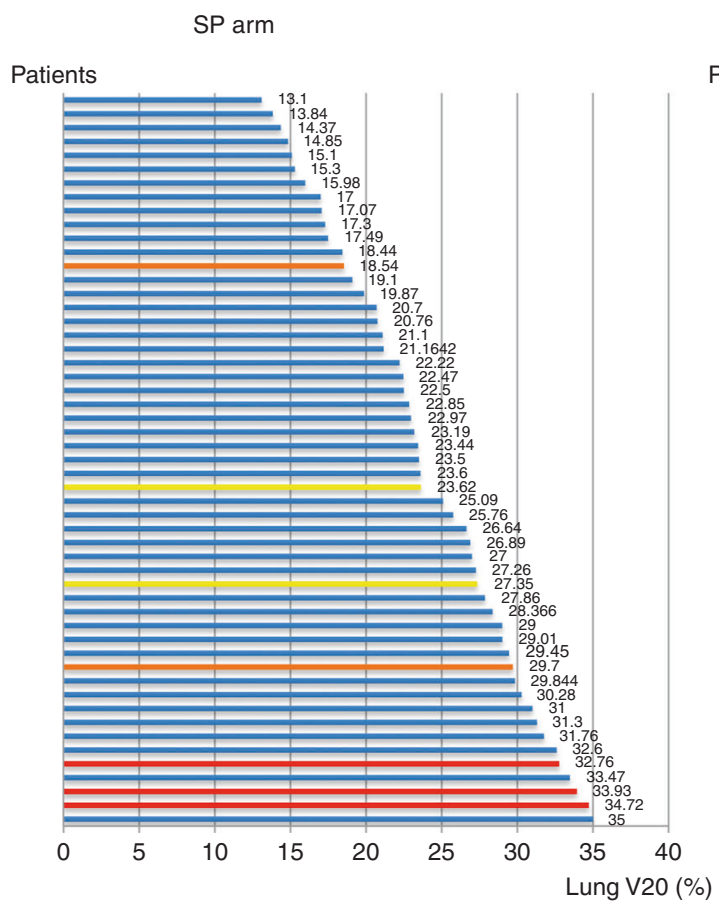

VP arm
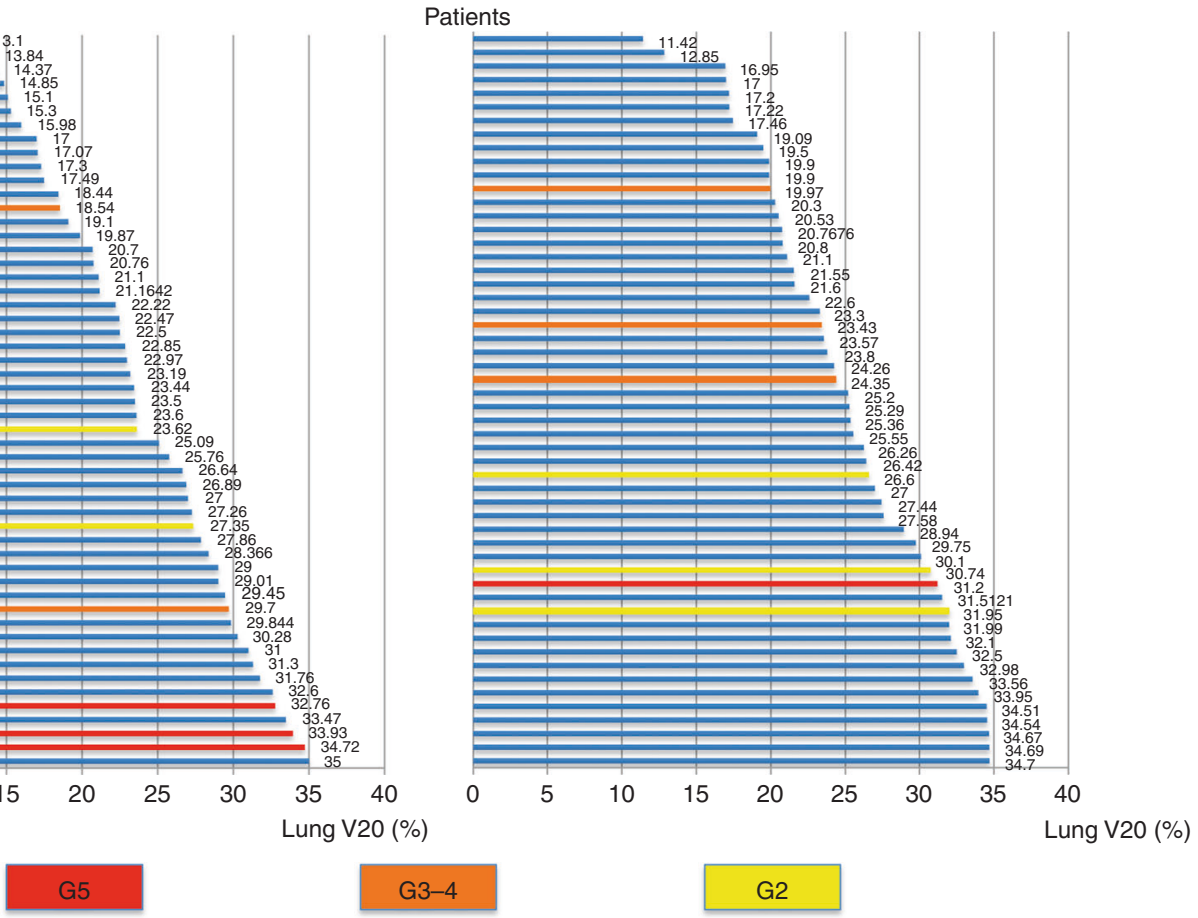

G3-4

G2

Fig. 2 Relationship between lung V20 and radiation pneumonitis. The relationship between lung V20 and pneumonitis grading in the SP arm (S-1 plus cisplatin plus thoracic radiotherapy) and the VP arm (vinorelbine plus cisplatin plus thoracic radiotherapy). The patients are arranged in ascending order of V20 on the vertical axis and lung V20 is plotted on the horizontal axis; the grade of pneumonitis is colour coded

higher than expected, while the QA results from radiotherapy did not indicate that deviations were directly associated with TRDs. Thus, we need to review exclusion criteria for radiotherapy to provide safer treatment for patients with LA-NSCLC in the future trial.

The present study also adopted the regimen used in WJTOG3706 for the SP arm. Ohyanagi et al..$^{9}$ and Kaira et al. ${ }^{10}$ have also conducted phase II studies using SP regimens and the efficacy of the SP regimen used in the present study is similar to that reported in their studies; the toxicity is considered acceptable. Various VP regimens have been examined (i.e. no radiation in the first cycle and concurrent radiotherapy in the second and third cycles with additional chemotherapy in the fourth cycle, or oral administration of vinorelbine) and no treatment strategy has been established for this regimen. $6,711,12$ Induction chemotherapy is no longer considered to be the standard of care for lung cancer. We thus adopted the regimen proposed by Sekine et al., ${ }^{15}$ which is the most common chemoradiotherapeutic regimen used in Japan. Sekine et al. ${ }^{8,15}$ used 1 -week cessation of radiation during treatment. However, recent radiotherapy is, in principle, given without cessation and additional adjuvant chemotherapy generally uses the same regimen as that used in concurrent radiotherapy. Therefore, radiotherapy was continued without cessation in this study and the regimen prescribed for concurrent radiotherapy was used instead of docetaxel during the consolidation phase. Although the efficacy of the VP regimen studied was similar to those reported in previous studies, toxicity was higher than that expected and higher than that observed with other regimens using vinorelbine. However, we consider the toxicity of our VP regimen to be acceptable, suggesting that the regimen used in the present study could become a standard VP regimen.

When these results are compared with those from previous phase III studies, 2-year PFS is equal to that reported in PROCLAIM, RTOG0617, and WJTOG0105 and toxicity is acceptable, except for a slightly higher incidence of TRDs; ${ }^{3,13,14}$ both the SP and VP regimens should be fully evaluated as potential treatments in future studies.

There was no difference in the treatment completion rate between the VP arm and the SP arm during the consolidation phase, although the VP arm had a lower treatment completion rate than the SP arm during the concurrent phase for several reasons including delayed treatment and discontinuation due to haematological toxicity. The treatment completion rate in the SP arm was similar to that reported in WTOG3706 and other phase II studies, and the obtained results may be reproducible. Because there were variations in previous VP regimens, this made direct comparison somewhat difficult. ${ }^{6-8,11}$ However, haematological toxicity tended to be higher in the VP arm than in the SP arm and treatment suspension and dose reduction were required. Although efficacy and toxicity appeared to be similar in the two arms, the SP arm tended to have slightly more favourable OS results in every subgroup, and PFS in the SP arm also appeared to be superior to that in the VP arm for a long-term course of treatment (Fig. 3a). This may be due to the fact that the SP arm has a slightly better outcome in terms of local control and treatment compliance.

The patterns of first failure by site were different between the arms. A preceding phase II study (WJTOG3706) also showed that distant failure was more frequent than local failure $(68 \%$ versus $32 \%){ }^{5}$ Other previous studies have also reported that the incidence of recurrence within the radiation field tended to be low in the SP regimen and no specific tendency was observed for the VP regimen. ${ }^{9,10,15,16}$ The risk of local recurrence may be reduced by continuous administration of $\mathrm{S}-1$ acting as a radiosensitizer in the SP regimen. In contrast, the frequency of distant recurrence is high, and it may be necessary to evaluate additional regimens which are more effective against distant metastases.

More TRDs occurred in this trial than were expected, although all of the adverse events reported in this series can occur with TRT 


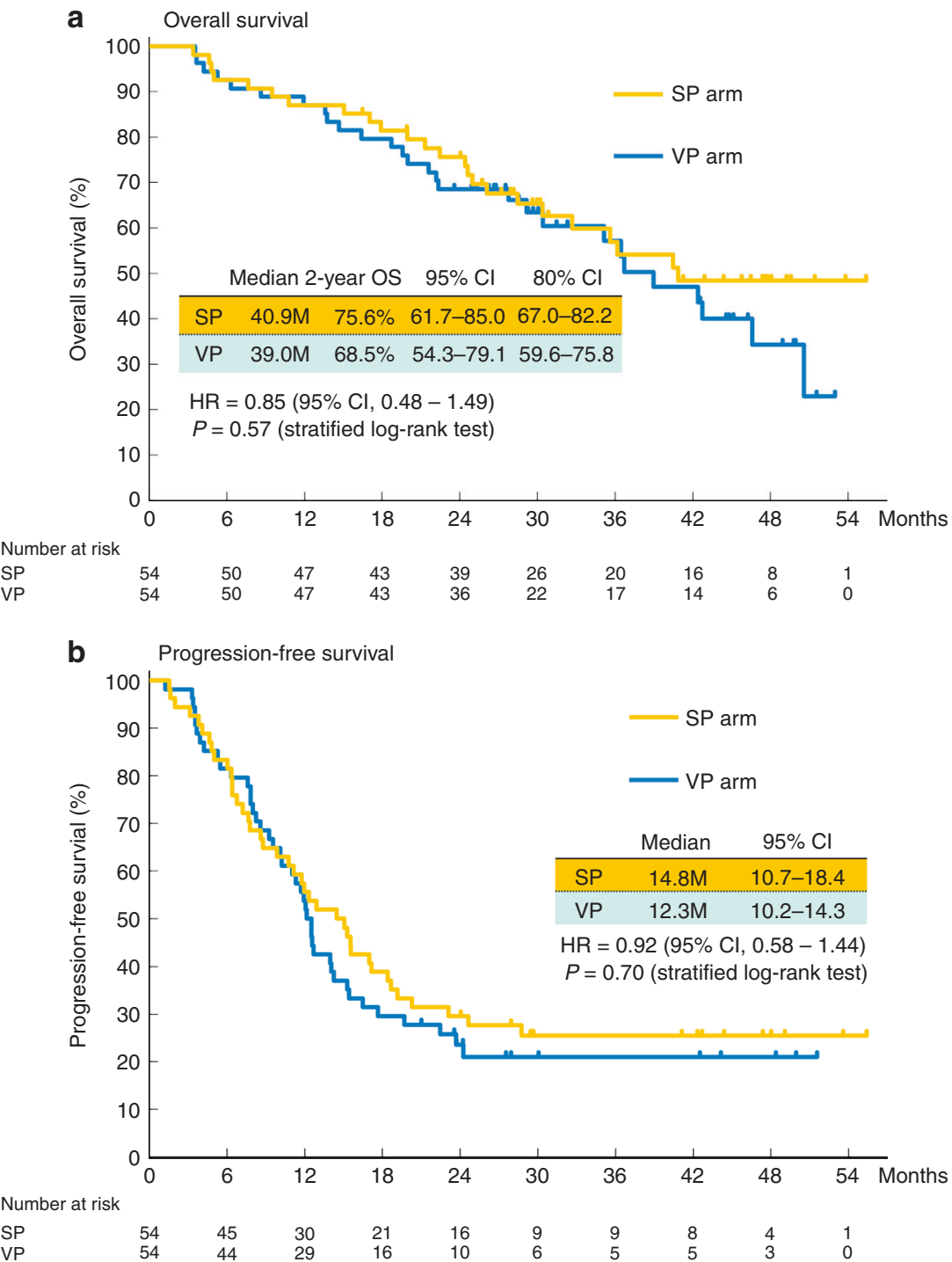

Fig. 3 Survival curves. Kaplan-Meier estimates of a overall survival (OS; primary endpoint) in the full analysis set (FAS) and $\mathbf{b}$ progression-free survival (PFS) in the FAS. OS was measured from the date of random assignment to the date of death as a result of any cause. At the cutoff date for data inclusion in the analysis, if a patient had not died, OS was censored at the last date they were known to still be alive. PFS was measured from the date of random assignment to the first date of documented objective progression of disease, or of death as a result of any cause. PFS was censored at the date of the last objective progression-free disease assessment before the date of any subsequent systemic anticancer therapy or death, whichever applied first. Cl confidence interval, SP arm S-1 plus cisplatin plus thoracic radiotherapy, VP arm vinorelbine plus cisplatin plus thoracic radiotherapy

for lung cancer. A previous phase $1 / I$ trial for patients with lung cancer treated with combination TRT and SP regimens or VP regimens showed a TRD rate of $<5 \% ., 5-12,16-21$

Three patients in the SP arm and one patient in the VP arm died of radiation pneumonitis. Palma et al. ${ }^{22}$ reported that fatal pneumonitis, although uncommon, is related to dosimetric factors such as V20 and mean lung dose. In the present study, the frequency of radiation pneumonitis (grade 2 or greater) was significantly increased in patients with a V20 $\geq 30 \%$, which is also consistent with the report. Although previous studies (PROCLAIM, RTOG0617, KCSG-LU05-04) have reported that the risk of TRDs, including fatal radiation pneumonitis and pulmonary haemorrhage, is $3 \%$ to $5 \%$, the rate was relatively high in the present study at $8 \%{ }^{13,14,23}$ QA results from radiotherapy in all patients indicated that there were no TRDs caused by deviations from the protocol. There was no significant relationship between V20 >30\% and radiation pneumonitis grade 5, although the lung V20 exceeded $30 \%$ in patients in the SP arm who died of radiation pneumonitis. Regarding pulmonary doses (V20), regulatory controls should be stricter in future clinical trials

The present study has the following limitations: (1) as with previous studies, the significance of consolidation is not clear; (2) the VP regimen adopted may not have been optimal; and (3) the study population was exclusively Japanese and the obtained data cannot be regarded as globally applicable.

Although the present study found no significant difference between the SP arm and the VP arm, the PFS curve showed more favourable results for the SP arm over the long term, and treatment compliance was better in the SP arm than in the VP arm. After a comprehensive consideration of these results, WJOG therefore intends to study the SP arm in the next phase. However, results for adjuvant treatment with durvalumab after definitive chemoradiotherapy for patients with LA-NSCLC have been reported recently. ${ }^{24}$ Consolidation chemotherapy using an immune checkpoint inhibitor may become standard treatment in the near future. Therefore, we have to evaluate the feasibility of 


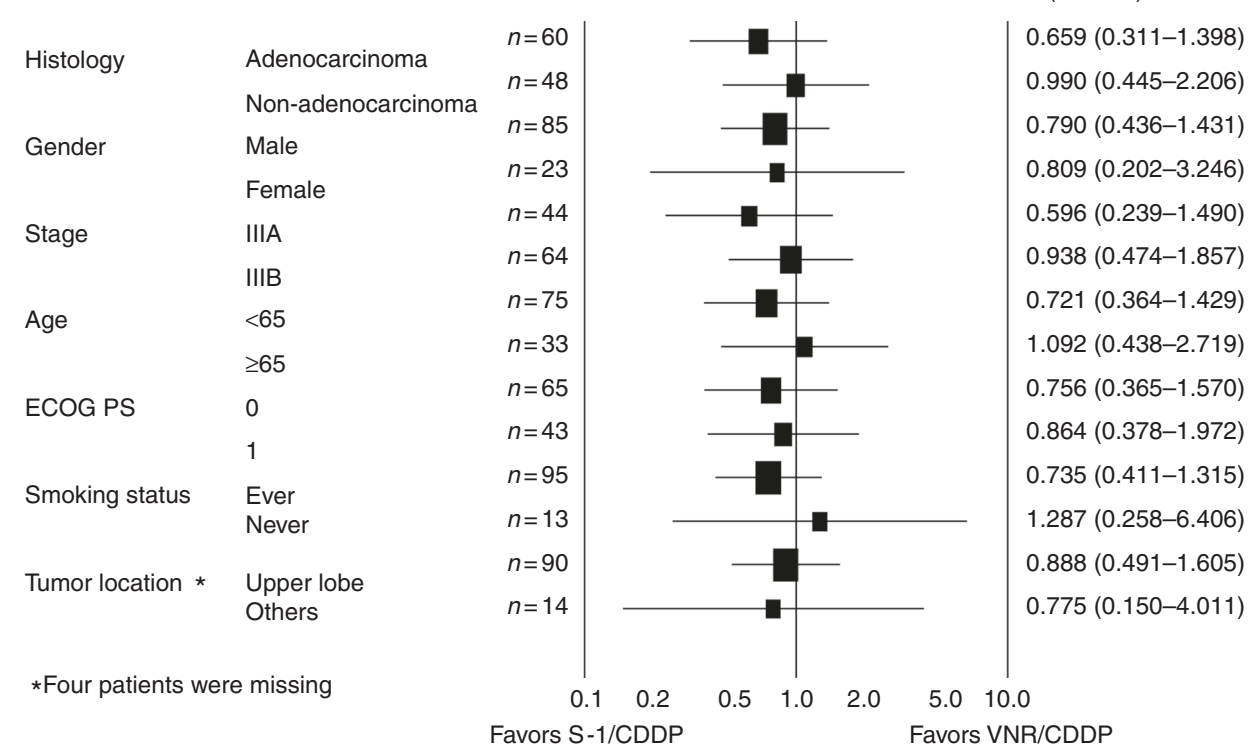

Fig. 4 Subset analysis. Overall survival hazard ratio in subgroups according to baseline characteristics. Cl confidence interval, ECOG PS Eastern Cooperative Oncology Group performance status, CDDP cisplatin, VNR vinorelbine

consolidation chemotherapy using durvalumab after TRT with concurrent S-1 plus cisplatin while carefully considering the risk of pulmonary toxicity caused by radiotherapy before initiation of the phase III trial.

\section{ACKNOWLEDGEMENTS}

We would like to express special thanks to all participating patients and their families, the Data and Safety Monitoring Committee, the Audit Committee of WJOG, and the WJOG Data Center. This trial was funded by TAIHO Pharmaceutical Co., Ltd., Japan under a research contract. We acknowledge the following 23 participating institutions: Cancer Institute Hospital of JFCR, Shizuoka Cancer Center, Kanagawa Cancer Center, Aichi Cancer Center Hospital, Aichi Cancer Center Aichi Hospital, National Hospital Organization Nagoya Medical Center, Gifu Municipal Hospital, Kindai University Hospital, Faculty of Medicine, Osaka City University Hospital, Osaka General Medical Center, Osaka Medical Center for Cancer and Cardiovascular Diseases, Osaka Prefectural Medical Center for Respiratory and Allergic Diseases, Osaka City General Hospital, Izumi Municipal Hospital, Osaka Saiseikai Nakatsu Hospital, Hyogo Cancer Center, Institute of Biomedical Research and Innovation Hospital, Hiroshima City Hiroshima Citizens Hospital, Tottori University hospital, National Hospital Organization Shikoku Cancer Center, Kyushu University Hospital, National Hospital Organization Kyushu Cancer Center, and lizuka Hospital.

\section{AUTHOR CONTRIBUTIONS}

Conception and design: T. Seto, T. Sasaki, Y.I., K.N., Y. Nishimura, Y. Nakanishi. Administrative support: H.H., N. Yamamoto. Provision of study materials or patients: T. Sasaki, T. Seto, N.K., J.S., T. Kodaira, M.N., T. Kozuka, T.T., H.H., N. Yoshimura, S.T., H.K., M.K. Collection and assembly of data: T. Sasaki, T. Seto, T.Y., N.K., J.S., T. Kodaira, M.N., T. Kozuka, T.T., H.K., N. Yoshimura, S. T., H.K., M.K., Y.I., K. Nakagawa, Y. Nishimura, N. Yamamoto, Y.Nakanishi. Data analysis and interpretation: T. Sasaki, T. Seto, T.Y., K. Nakagawa, Y. Nishimura, N. Yamamoto, Y. Nakanishi. Manuscript writing: T. Sasaki, T. Seto, T.Y. Final approval of manuscript: T. Sasaki, T. Seto, T.Y., N.K., J.S., T. Kodaira., M.N., T. Kozuka, T.T., H.K., N. Yoshimura, S.T., H.K., M.K., Y.I., K. Nakagawa, Y. Nishimura, N. Yamamoto, Y. Nakanishi

\section{ADDITIONAL INFORMATION}

Supplementary information is available for this paper at https://doi.org/10.1038/ s41416-018-0243-2.

Competing interests: All authors had full access to all the data in the study and take responsibility for the integrity of the data and the accuracy of the data analysis. T. Seto has received honoraria from Kyowa Hakko Kirin Co., Ltd. and Taiho
Pharmaceutical Co., Ltd. T.Y. has received personal fees from Taiho Pharmaceutical Co., Ltd. N.K. has received personal fees from Taiho Pharmaceutical Co., Ltd. M.N. has received grants and personal fees from Taiho Pharmaceutical Co., Ltd. T.T. has received grants and personal fees from Taiho Pharmaceutical Co., Ltd. Y.I. has received honoraria from Kyowa Hakko Kirin Co., Ltd. and Taiho Pharmaceutical Co., Ltd. K.N. has received grants and personal fees from Taiho Pharmaceutical Co., Ltd., during the conduct of the study; grants and personal fees from Kyowa Hakko Kirin Co., Ltd. N. Yamamoto has received grants and personal fees from Kyowa Hakko Kirin Co., Ltd. and Taiho Pharmaceutical Co., Ltd. Y. Nakanishi has received grants from Taiho Pharmaceutical Co., Ltd. All other authors declared no competing interests.

Ethics approval and consent to participate: Each center's institutional review board or ethics committee approved the study. The trial followed the principles of the Declaration of Helsinki and the Japanese Ethical Guidelines for Clinical Research. All patients provided written informed consent including the publication.

Consent for publication: All authors gave final approval of the version to be published.

Availability of data and material: All data are available via the corresponding author and the WJOG.

Funding: This work was supported by West Japan Oncology Group (WJOG). The Research fund was provided to WJOG by Taiho Pharmaceutical Co. Ltd under a research contract. The funders did not have any involvement in the design of the study; the collection, analysis, and interpretation of the data; the writing of the article; or the decision to submit the article for publication. No grant numbers are applicable.

\section{REFERENCES}

1. Torre, L. A. et al. Global cancer statistics, 2012. CA Cancer J. Clin. 65, 87-108 (2015).

2. Segawa, Y. et al. Phase III trial comparing docetaxel and cisplatin combination chemotherapy with mitomycin, vindesine, and cisplatin combination chemotherapy with concurrent thoracic radiotherapy in locally advanced non-smallcell lung cancer: OLCSG 0007. J. Clin. Oncol. 28, 3299-3306 (2010).

3. Yamamoto, N. et al. Phase III study comparing second- and third-generation regimens with concurrent thoracic radiotherapy in patients with unresectable stage III non-small-cell lung cancer: West Japan Thoracic Oncology Group WJTOG0105. J. Clin. Oncol. 28, 3739-3745 (2010).

4. Shirasaka, T. et al. Antitumor activity of $1 \mathrm{M}$ tegafur-0.4 M 5-chloro-2,4-dihydroxypyridine-1 $\mathrm{M}$ potassium oxonate (S-1) against human colon carcinoma orthotopically implanted into nude rats. Cancer Res. 56, 2602-2606 (1996). 
5. Ichinose, Y. et al. S-1 plus cisplatin with concurrent radiotherapy for locally advanced non-small cell lung cancer: a multi-institutional phase II trial (West Japan Thoracic Oncology Group 3706). J. Thorac. Oncol. 6, 2069-2075 (2011).

6. Vokes, E. E. et al. Randomized phase II study of cisplatin with gemcitabine or paclitaxel or vinorelbine as induction chemotherapy followed by concomitant chemoradiotherapy for stage IIIB non-small-cell lung cancer: cancer and leukemia group B study 9431. J. Clin. Oncol. 20, 4191-4198 (2002).

7. Zatloukal, P. et al. Concurrent versus sequential chemoradiotherapy with cisplatin and vinorelbine in locally advanced non-small cell lung cancer: a randomized study. Lung Cancer 46, 87-98 (2004).

8. Sekine, I. et al. Docetaxel consolidation therapy following cisplatin, vinorelbine, and concurrent thoracic radiotherapy in patients with unresectable stage III nonsmall cell lung cancer. J. Thorac. Oncol. 1, 810-815 (2006).

9. Ohyanagi, F. et al. Phase II trial of S-1 and cisplatin with concurrent radiotherapy for locally advanced non-small-cell lung cancer. Br. J. Cancer 101, 225-231 (2009).

10. Kaira, K. et al. Phase II study of oral S-1 and cisplatin with concurrent radiotherapy for locally advanced non-small-cell lung cancer. Lung Cancer 82, 449-454 (2013).

11. Krzakowski, M. et al. Oral vinorelbine and cisplatin as induction chemotherapy and concomitant chemo-radiotherapy in stage III non-small cell lung cancer: final results of an international phase II trial. J. Thorac. Oncol. 3, 994-1002 (2008).

12. Juan, O. et al. Full-dose cisplatin and oral vinorelbine concomitant with radiotherapy in unresectable stage III non-small cell lung cancer: a multi-center phase II study. Anticancer Res. 34, 1959-1966 (2014).

13. Senan, S. et al. PROCLAIM: randomized phase III trial of pemetrexed-cisplatin or etoposide-cisplatin plus thoracic radiation therapy followed by consolidation chemotherapy in locally advanced nonsquamous non-small-cell lung cancer. $J$. Clin. Oncol. 34, 953-962 (2016).

14. Bradley, J. D. et al. Standard-dose versus high-dose conformal radiotherapy with concurrent and consolidation carboplatin plus paclitaxel with or without cetuximab for patients with stage IIIA or IIIB non-small-cell lung cancer (RTOG 0617): a randomised, two-by-two factorial phase 3 study. Lancet Oncol. 16, 187-199 (2015).

15. Sekine, I. et al. Phase I study of cisplatin, vinorelbine, and concurrent thoracic radiotherapy for unresectable stage III non-small cell lung cancer. Cancer Sci. $\mathbf{9 5}$, 691-695 (2004).

16. Horinouchi, $\mathrm{H}$. et al. Long-term results of concurrent chemoradiotherapy using cisplatin and vinorelbine for stage III non-small-cell lung cancer. Cancer Sci. 104, 93-97 (2013).
17. Shukuya, T. et al. Comparison of vinorelbine plus cisplatin and $S-1$ plus cisplatin in concurrent chemoradiotherapeutic regimens for unresectable stage III non-small cell lung cancer. Anticancer Res. 32, 675-680 (2012).

18. Yao, L. et al. S-1 plus cisplatin with concurrent radiotherapy versus cisplatin alone with concurrent radiotherapy for stage III non-small cell lung cancer: a pilot randomized controlled trial. Radiat. Oncol. 10, 10 (2015).

19. Nogami, N. et al. A phase II study of cisplatin plus S-1 with concurrent thoracic radiotherapy for locally advanced non-small-cell lung cancer: The Okayama Lung Cancer Study Group Trial 0501. Lung Cancer 87, 141-147 (2015).

20. Hirose, $T$. et al. The combination of cisplatin and vinorelbine with concurrent thoracic radiation therapy for locally advanced stage IIIA or IIIB non-small-cell lung cancer. Cancer Chemother. Pharmacol. 58, 361-367 (2005).

21. Imamura, F. et al. Novel chemoradiotherapy with concomitant boost thoracic radiation and concurrent cisplatin and vinorelbine for stage IIIA and IIIB nonsmall-cell lung cancer. Clin. Lung Cancer 15, 281-286 (2014).

22. Palma, D. A. et al. Predicting radiation pneumonitis after chemoradiation therapy for lung cancer: an international individual patient data meta-analysis. Int. J. Radiat. Oncol. Biol. Phys. 85, 444-450 (2013).

23. Ahn, J. S. et al. Multinational randomized phase III trial with or without consolidation chemotherapy using docetaxel and cisplatin after concurrent chemoradiation in inoperable stage III non-small-cell lung cancer: KCSG-LU05-04. J. Clin. Oncol. 33, 2660-2666 (2015).

24. Antonia, S. J. et al. Durvalumab after chemoradiotherapy in stage III non-smallcell lung cancer. N. Engl. J. Med. 377, 1919-1929 (2017).

(c) (i) Open Access This article is licensed under a Creative Commons By Attribution 4.0 International License, which permits use, sharing, adaptation, distribution and reproduction in any medium or format, as long as you give appropriate credit to the original author(s) and the source, provide a link to the Creative Commons license, and indicate if changes were made. The images or other third party material in this article are included in the article's Creative Commons license, unless indicated otherwise in a credit line to the material. If material is not included in the article's Creative Commons license and your intended use is not permitted by statutory regulation or exceeds the permitted use, you will need to obtain permission directly from the copyright holder. To view a copy of this license, visit http://creativecommons. org/licenses/by/4.0/.

(c) The Author(s) 2018 